\title{
Staged lower lip reconstruction following gangrenous stomatitis in an immunosuppressed patient
}

\author{
Han Byeol Jin, \\ Jeong Yeol Yang, \\ Kyung Sik Kim, \\ Seung Hong Kim, \\ Joon Choe, \\ Jee Hyeok Chung \\ Department of Plastic and \\ Reconstructive Surgery, Myongji \\ Hospital, Goyang, Korea
}

\begin{abstract}
A 70-year-old male with a history of diabetes mellitus, hypertension, and coronary stent insertion visited our hospital 7 days after biting his lower lip. Swelling and inflammation had worsened despite debridement and antibiotic treatment. On the 8th hospital day, fungal infection with Candida albicans and superimposed bacterial infection with Klebsiella pneumoniae were found on tissue culture. Extensive necrosis resulted in a defect of approximately $3 / 4$ of the entire lower lip and a full-layer skin defect from the vermilion to the gingivobuccal sulcus at the right corner of the mouth. To correct drooling, incomplete lip sealing, and trismus, staged reconstruction was performed with consideration of cosmetic and functional features. The treatment process using staged reconstruction and antifungal treatment for an extensive lower lip defect caused by fungal stomatitis is described.
\end{abstract}

Keywords: Stomatitis / Candida / Gangrene

\section{INTRODUCTION}

Gangrenous stomatitis has generally been thought of as associated with infection by Fusobacterium species, Prevotella species, and similar agents $[1,2]$. It is known to develop in children with poor nutritional status in developing countries and causes severe soft tissue deformities of the face and facial bones [3-6]. However, some cases of gangrenous stomatitis similar to noma have been reported in adult patients without malnutrition or previous bacterial infection $[7,8]$. Most of these patients had an immunocompromised condition such as diabetes mellitus or acquired immunodeficiency syndrome. The authors treated a case of gangrenous stomatitis caused by an opportunistic fungal infection in a patient with diabetes mellitus. This presentation is a review of the treatment process in a patient with gangrenous stomatitis using antifungal medication, surgical debridement, and staged reconstruction.

\section{Correspondence: Jeong Yeol Yang}

Department of Plastic and Reconstructive Surgery, Myongji Hospital, 55 Hwasu-ro

14beon-gil, Deokyang-gu, Goyang 10475, Korea

E-mail: jyyang2@mjh.or.kr

Received May 30, 2018 / Revised July 30, 2018 / Accepted August 6, 2018

\section{CASE REPORT}

A 70-year-old male with a history of diabetes mellitus, hypertension, and coronary stent insertion visited Myongji Hospital 7 days after biting his lower lip. Swelling and inflammation had worsened, and several ulcerative mucosal lesions and purulent discharge with foul odor were observed on the lower lip. The patient was admitted for high-dose intravenous antibiotic treatment and serial debridement following demarcation of the wound.

At the time of admission, the patient had a serum glucose level of $527 \mathrm{mg} / \mathrm{dL}$, hemoglobin A1C of 11.1\%, white blood cell

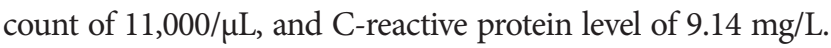
We used a beta-lactamase resistant penicillin and a quinolone as intravenous antibiotics and irrigated the oral cavity with povidone iodine and benzethonium chloride solution three to four times a day. On the 2nd hospital day, the patient's lower lip appeared gangrenous, and the C-reactive protein level was $18.67 \mathrm{mg} / \mathrm{dL}$. Facial computed tomography showed diffuse cellulitis with gangrenous changes and abscess formation involving the lower lip (Fig. 1). Surgical debridement was performed 

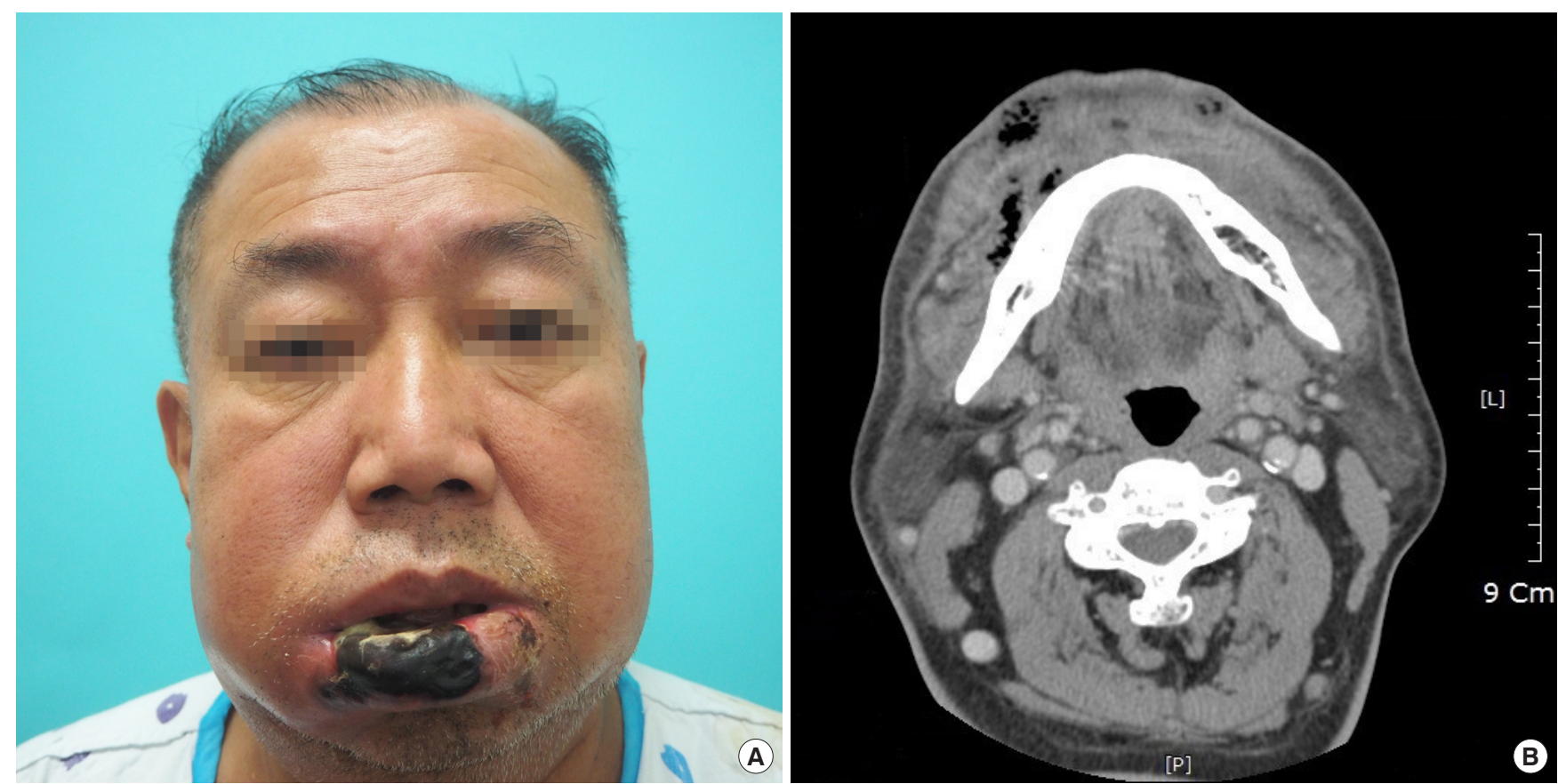

Fig. 1. (A) A 70-year-old male with diabetes mellitus and gangrenous changes in the lower lip with severe infection. (B) Facial computed tomography showing diffuse cellulitis with abscess formation in the lower lip.

under local anesthesia on the 4th hospital day. Debridement involved $3 / 4$ of the lower lip vermilion, extending to a $2.5 \times 1.5$ $\mathrm{cm}^{2}$ area of skin at the right corner of the mouth. Subsequent chemical ablation was performed on the raw surface of the debridement site using Albothyl solution, and a silastic drain was inserted. After the 1st surgical debridement, the C-reactive protein level decreased to $6.48 \mathrm{mg} / \mathrm{L}$ on the 5 th hospital day. However, the wound infection and inflammatory markers did not improve. More soft tissue of the lower lip became necrotic despite surgical debridement and continued antibiotic treatment.

On the 8th hospital day, fungal infection due to Candida albicans and superimposed bacterial infection due to Klebsiella pneumoniae were found on tissue culture performed at the time of admission. An antifungal drug (fluconazole $100 \mathrm{mg}$ once a day) was added and the antibiotics were changed from ampicillin/sulbactam $3 \mathrm{~g}$ four times a day and clindamycin $150 \mathrm{mg}$ four times a day to piperacillin/tazobactam $4.5 \mathrm{~g}$ three times a day. On the 11th hospital day, the C-reactive protein level was $0.75 \mathrm{mg} / \mathrm{dL}$, and signs of infection were much improved (Table 1). The decision was made to perform local flap coverage with surgical debridement. Under local anesthesia, vermilionectomy was performed on the lower lip, which had remaining debris. The defect was reconstructed using a local buccal mucosal flap. After the 2nd surgical debridement, we continued antibiotic and antifungal treatment and conservative wound dressings for 2 weeks. The C-reactive protein level gradually improved from $0.75 \mathrm{mg} / \mathrm{L}$ to $0.06 \mathrm{mg} / \mathrm{L}$. The gangrenous stomatitis was healed
Table 1. Treatment flowchart of this case

\begin{tabular}{|c|}
\hline Flowchart \\
\hline A 70-year-old male with diabetes mellitus and severe infection of the lower lip \\
\hline $\begin{array}{l}\rightarrow \text { Admission for high dose intravenous antibiotics, intensive wound management, } \\
\text { surgical debridement }\end{array}$ \\
\hline $\begin{array}{l}\rightarrow \text { On the 4th hospital day, no response to antibiotics, gangrenous change in the } \\
\quad \text { lower lip }\end{array}$ \\
\hline$\rightarrow$ Surgical debridement, chemical ablation with Albothyl solution \\
\hline$\rightarrow$ More soft tissue necrosis \\
\hline$\rightarrow$ On the 8th hospital day, fungal infection was identified on tissue culture \\
\hline$\rightarrow$ Added antifungal drug (fluconazole 100 mg once a day), change in antibiotics \\
\hline$\rightarrow$ Infection subsided, inflammatory markers normalized \\
\hline
\end{tabular}

by the 23rd hospital day.

The patient was followed for about 6 months. Extensive necrosis involved approximately $3 / 4$ of the entire lower lip, with a full-layer skin defect extending from the vermilion to the gingivobuccal sulcus at the right corner of the mouth. Because of extensive soft tissue destruction of the lower lip, the patient had functional and aesthetic defects, with trismus, incomplete mouth closure, persistent drooling, discontinuity of the lower lip, and tooth exposure (Fig. 2).

Six months later, Abbe flap coverage of the right corner of the mouth was performed to correct the drooling and trismus (Fig. 3). Three months after Abbe flap coverage, vermilion apron flap coverage of the central portion of the lower lip was performed to correct the drooling and strengthen lip sealing (Fig. 4). On the follow-up at the outpatient department, drooling from the 


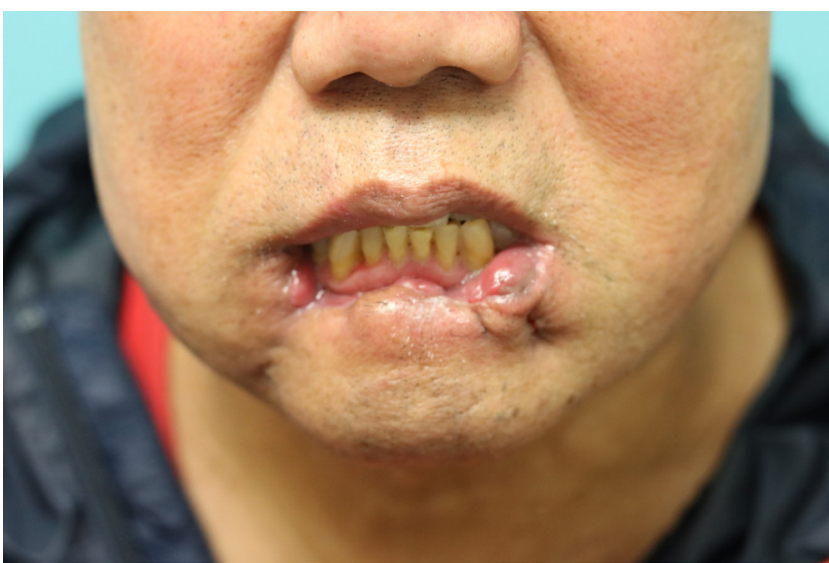

Fig. 2. As a result of extensive soft tissue necrosis caused by acute fungal stomatitis, 3/4 of the entire lower lip was affected, with a fulllayer skin defect extending from the vermilion to the gingivobuccal sulcus at the corner of the mouth.
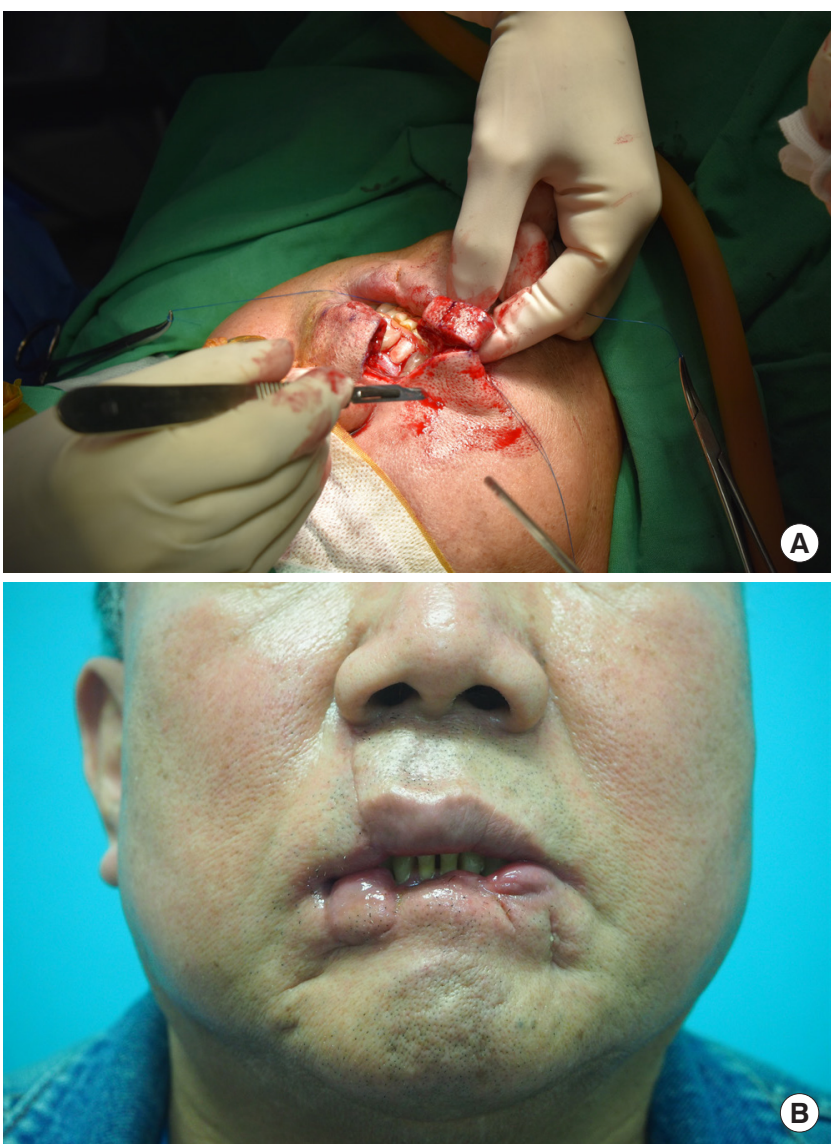

Fig. 3. (A) Six months after the acute fungal stomatitis resolved, Abbe flap coverage was performed to correct drooling at the right the corner of the mouth. (B) Result after Abbe flap coverage.

lower lip was functionally corrected, and the patient was satisfied with the cosmetic outcome (Fig. 5). Revision of the notching deformity and incisional scars of the lower lip was planned for a later time (Table 2).
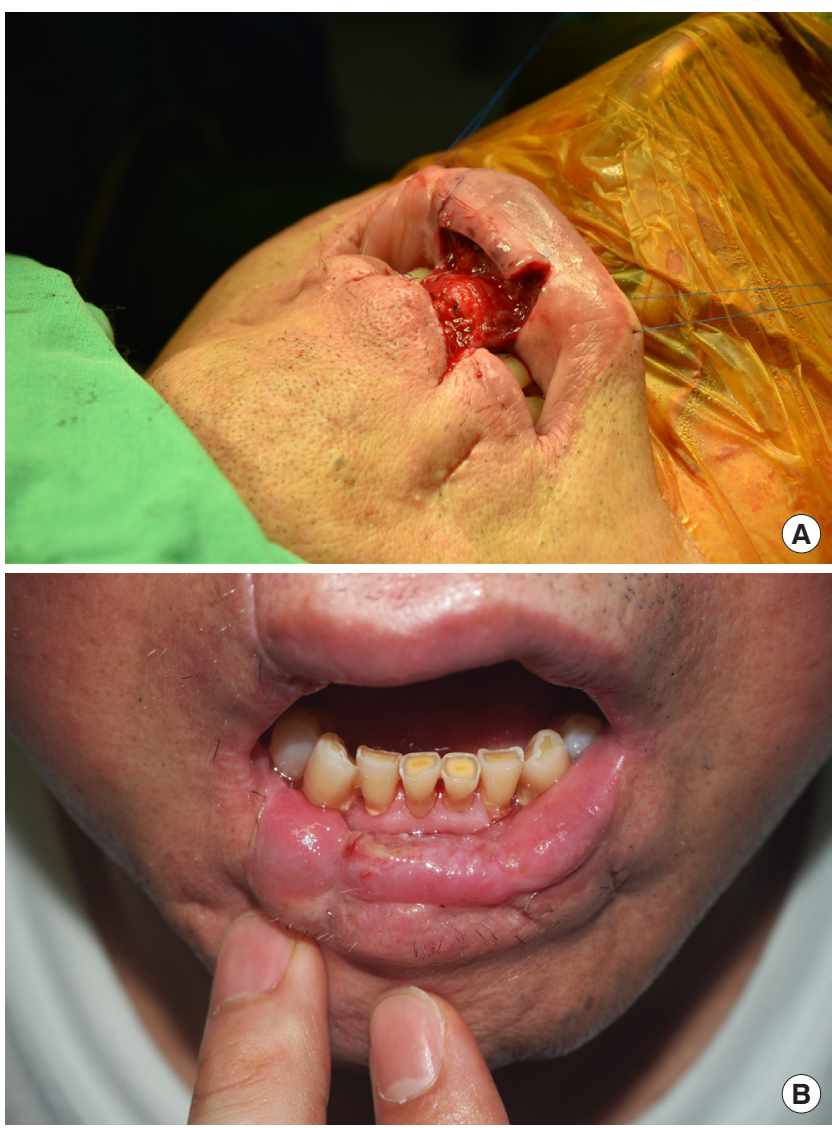

Fig. 4. (A) Three months after Abbe flap coverage, a vermilion apron flap was performed to correct the drooling and whistle deformity of the central lower lip. (B) Result after vermilion apron flap coverage.

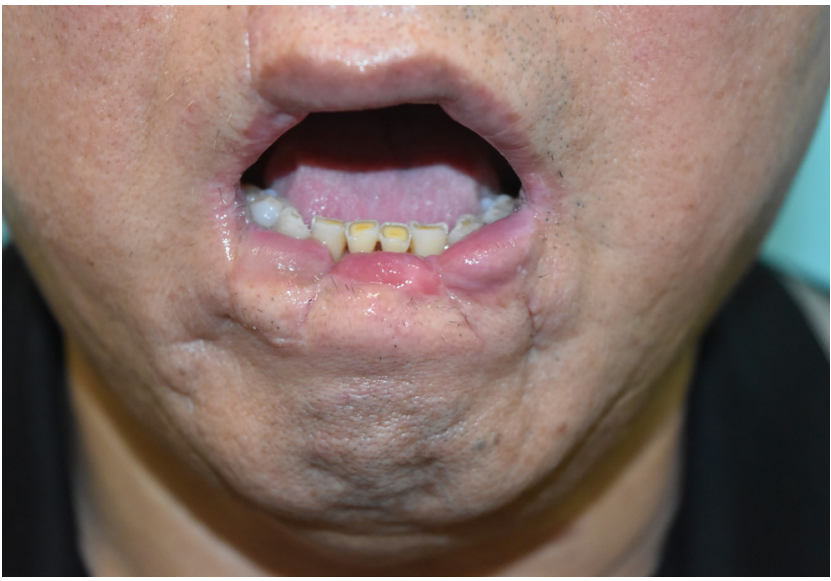

Fig. 5. Result at 6 months after surgery.

\section{DISCUSSION}

Gangrenous stomatitis is an infection of the oral cavity mainly caused by Fusobacterium species and Prevotella species [1,2]. These bacteria are pathogens involved in necrotizing stomatitis 
Table 2. Continued, treatment flowchart of this case

Flowchart
On the 11th hospital day, 2nd surgical debridement, vermilionectomy, local flap
coverage
$\rightarrow$ Recovered from acute fungal stomatitis
$\rightarrow$ As a result, $3 / 4$ of the entire lower lip and a full-layer skin defect extended from
the vermilion to the gingivobuccal sulcus at the corner of the mouth
$\rightarrow 6$ Months later, Abbe flap used for drooling at the corner of the mouth and
trismus
$\rightarrow 3$ Months later, vermilion apron flap coverage of the central lower lip for
reconstruction of vermilion, correction of whistle deformity, eliminating
drooling, strengthening lip sealing

in young adults. They promote tissue destruction through their ability to degrade lipids and produce proteolytic enzymes [6]. Malnourished patients have different flora from those of healthy persons, as reported by Sawyer and Nwoku [9]. The outcome of infection has been strikingly improved by the use of antibiotics, especially penicillin, and the mortality rate is now $8 \%-10 \%$ [4].

Gangrenous stomatitis is almost exclusively seen in young children living in remote areas of less developed countries, particularly in Africa. However, rare cases have been reported in adult patients from developed countries, especially in the presence of blood dyscrasias and immunocompromised states [10]. Stassen et al. [7] reported the unusual presentation of necrotizing stomatitis in a healthy white adult Caucasian woman in a part of the world where the disease is very uncommon. Moon et al. [8] reported facial deformities following necrotizing stomatitis in five patients aged between 47 and 58 years.

Our patient was a 70-year-old man with diabetes mellitus, hypertension, and coronary stent insertion. Patients with diabetes mellitus are in an immunocompromised state, as a result of two mechanisms. First, diabetic polymorphonuclear cells and monocytes/macrophages have decreased function (chemotaxis, phagocytosis, killing), and second, microorganisms show increased adherence to diabetic cells. C. albicans expresses a surface protein that has great homology with the receptor for complement factor 3b. In a hyperglycemic condition, expression of the receptor-like protein of C. albicans is increased, resulting in competitive binding and inhibition of complement-mediated phagocytosis [11]. Darwazeh et al. [12] recently showed a significant increase in the adhesion of Candida to oral epithelial cells in patients with diabetes, when compared with a healthy population.

Adhesion of Candida to epithelial cells, widely recognized as an essential step in the process of Candida colonization and subsequent infection, is significantly inhibited by fluconazole. Fluconazole is secreted in the saliva in high concentration. It is tempting to speculate that fluconazole may interfere with the synthesis or structure of Candida receptors in buccal epithelial cells. Candida colonizes mucocutaneous surfaces, which can be portals of entry into deeper tissues when host defense is compromised [11].

In our case, a small wound developed in the oral mucosa and Candida present in the patient's mouth may have infected deep soft tissue, resulting in extensive soft tissue injury. Early detection of fungal infection prior to wound culture and the early use of fluconazole might have reduced the extensive soft tissue injury. Furthermore, the outcome would have been better if the patient had visited the hospital earlier, before worsening of the infection. Further studies are needed to investigate the prevalence of stomatitis caused by oral fungal infections in patients with diabetes, as well as the efficacy of prophylactic antifungal agents.

This report described a case of gangrenous stomatitis due to a fungal opportunistic infection in a patient with diabetes mellitus. The resulting extensive soft tissue defect extending from the vermilion to the gingivobuccal sulcus caused drooling and trismus. Patients with diabetes mellitus are in an immunocompromised state, and should maintain oral hygiene, control blood glucose levels, and receive appropriate and timely wound care for even small oral lesions. Physicians should always be mindful of the possibility of oral fungal infections in patients with diabetes mellitus and should always treat these as highrisk oral infections.

\section{CONFLICT OF INTEREST}

No potential conflict of interest relevant to this article was reported.

\section{PATIENT CONSENT}

The patients provided written informed consent for the publication and the use of their images.

\section{REFERENCES}

1. Falkler WA Jr, Enwonwu CO, Idigbe EO. Microbiological understandings and mysteries of noma (cancrum oris). Oral Dis 1999;5:150-5.

2. Paster BJ, Falkler WA Jr, Enwonwu CO, Idigbe EO, Savage KO, Levanos VA, et al. Prevalent bacterial species and novel phylotypes in advanced noma lesions. J Clin Microbiol 2002;40:218791.

3. Enwonwu CO, Falkler WA Jr, Idigbe EO, Savage KO. Noma (cancrum oris): questions and answers. Oral Dis 1999;5:144-9. 
4. Tempest MN. Cancrum oris. Br J Surg 1966;53:949-69.

5. Lazarus D, Hudson DA. Cancrum oris: a 35-year retrospective study. S Afr Med J 1997;87:1379-82.

6. Enwonwu CO, Falkler WA Jr, Phillips RS. Noma (cancrum oris). Lancet 2006;368:147-56.

7. Stassen LF, Batchelor AG, Rennie JS, Moos KF. Cancrum oris in an adult Caucasian female. Br J Oral Maxillofac Surg 1989;27: 417-22.

8. Moon JW, Lee SC, Cheon JS, Yang JY. Reconstruction of the acquired facial deformity due to cancrum oris sequelae. J Korean Soc Plast Reconstr Surg 2006;33:359-66.
9. Sawyer DR, Nwoku AL. Cancrum oris (noma): past and present. ASDC J Dent Child 1981;48:138-41.

10. Eisele DW, Inglis AF Jr, Richardson MA. Noma and noma neonatorum. Ear Nose Throat J 1990;69:119-20.

11. Scully C, el-Kabir M, Samaranayake LP. Candida and oral candidosis: a review. Crit Rev Oral Biol Med 1994;5:125-57.

12. Darwazeh AM, Lamey PJ, Lewis MA, Samaranayake LP. Systemic fluconazole therapy and in vitro adhesion of Candida albicans to human buccal epithelial cells. J Oral Pathol Med 1991;20:17-9. 